\title{
High Triglyceride Glucose Index Does Not Show the Presence and Severity of Coronary Artery Disease: A Single- Center Study
}

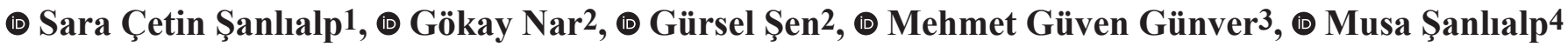

\author{
${ }^{1}$ Servergazi State Hospital, Clinic of Cardiology, Denizli, Turkey \\ 2Pamukkale University Faculty of Medicine, Department of Cardiology, Denizli, Turkey \\ 3̇istanbul University Faculty of Medicine, Department of Cardiology, İstanbul, Turkey \\ ${ }^{4}$ Denizli State Hospital, Clinic of Cardiology, Denizli, Turkey
}

\begin{abstract}
Objectives: The triglyceride glucose index (TyG index) calculated by fasting plasma glucose and triglycerides is associated with hypertension, diabetes and metabolic syndrome (MetS). However, the studies investigating the role of the TyG index in coronary artery disease (CAD) are limited and conflicting. In this study, we aimed to determine the role of the TyG index in the presence and severity of CAD in patients with acute coronary syndrome (ACS).

Materials and Methods: Eighty-three ACS patients (mean age: $64.64 \pm 12.79$ years; male gender, $65 \%$ ) and 50 control subjects with normal coronary arteries $(58.50 \pm 12.28$ years, male, $56 \%)$ were analyzed, retrospectively. The severity of CAD was evaluated by Gensini and SYNTAX I scores and $p<0.05$ was considered statistically significant.

Results: The TyG index did not differ significantly between ACS patients and controls $(8.97 \pm 0.72$ vs. 9.06 \pm 0.72 , $\mathrm{p}=0.533$ ). There was no correlation between the TyG index with SYNTAX and Gensini scores. In addition, TyG index was similar in subgroup analysis of ACS patients $(\mathrm{p}>0.05)$. When the subjects were divided according to the TyG index, those with high TyG index had a higher incidence of hypertension, diabetes, and lipid parameters deterioration $(\mathrm{p}<0.05)$.

Conclusion: TyG index does not indicate the presence and severity of CAD but may be closely related to dysmetabolic conditions that predispose to CAD.
\end{abstract}

Keywords: Cardiometabolic disorders, coronary artery disease, insulin resistance, triglyceride glucose index

Address for Correspondence: Sara Çetin Şanlıalp, Servergazi State Hospital, Clinic of Cardiology, Denizli, Turkey e-mail: saracetin@hotmail.com.tr ORCID: orcid.org/0000-0001-9328-9197

Received: 05.01.2021 Accepted: 29.03.2021

Cite this article as: : Çetin Şanlıalp S, Nar G, Şen G, Günver MG, Şanlıalp M. High Triglyceride Glucose Index Does Not Show the Presence and the Severity of Coronary Artery Disease: A Single-Center Study. EJCM 2021;9(2):76-82.

DOI: 10.32596/ejcm.galenos.2021-01-03

${ }^{\circ}$ Copyright 2021 by Heart and Health Foundation of Turkey (TÜSAV) / E Journal of Cardiovascular Medicine published by Galenos Publishing House. 


\section{Introduction}

Cardiovascular disease (CVD) is still among the leading causes of morbidity and mortality ${ }^{(1)}$. One of the factors that play an important role in CVD is insulin resistance (IR) related to cardiovascular risk factors such as hyperglycemia, dyslipidemia, and hypertension. The studies investigating the effects of IR on CVD have claimed that IR may accelerate atherogenesis by vascular occlusion, inflammation and thrombosis due to the disruption of insulin signal at the cellular level even in the absence of hyperglycemia ${ }^{(2)}$.

Today, a simple and inexpensive approach, the triglyceride glucose index (TyG index) derived from fasting plasma glucose and triglycerides is preferred for indirect evaluation of $\mathrm{IR}^{(3)}$. Recent studies have shown that the TyG index is associated with metabolic syndrome (MetS), arterial stiffness, carotid atherosclerosis, and coronary artery calcification (CAC) $)^{(4)}$. However, limited studies have reported the association of the TyG index with the presence and severity of CAD. Therefore, we aimed to evaluate the role of the TyG index in the diagnosis and progression of CAD in this study.

\section{Materials and Methods}

\section{Study Population}

In this retrospective study, we reviewed the medical records of 227 consecutive patients who underwent coronary angiography in Pamukkale University Cardiology Department between March 2020 and August 2020. The subjects diagnosed with acute coronary syndrome (ACS) consisting of ST-elevation myocardial infarction (STEMI), non-ST-elevation myocardial infarction (NSTEMI) and unstable angina pectoris (UA) were included in the patient group. The subjects with a history of angina or equivalent symptoms or a history of positive/suspicious stress test but showing normal coronary arteries during coronary angiography were determined as the control group.

Cardiomyopathies, cerebrovascular diseases, acute or chronic infections, malignancies, autoimmune diseases, severe kidney disfunction and severe liver disease were defined as exclusion criteria and finally, the study was performed with 133 subjects.

This study was approved by our institutional ethics committee in accordance with the Declaration of Helsinki (Pamukkale University Ethics Committee, date: 27/10/2020, protocol no: 020/70506). The requirement for informed consent was waived due to the retrospective design of the study.

The basic characteristics of the subjects such as age, gender, smoking, medication and laboratory parameters were analyzed retrospectively. The records obtained from fasting venous blood samples including glucose, total cholesterol, triglyceride (TG), low-density lipoprotein cholesterol (LDL-C), high-density lipoprotein cholesterol (HDL-C), creatinine, C-reactive protein (CRP) and hemogram parameters were examined. The TyG index was calculated by the formula Ln [fasting triglycerides $(\mathrm{mg} / \mathrm{dL}) \quad \mathrm{x}$ fasting blood glucose $(\mathrm{mg} / \mathrm{dL}) / 2]^{(5)}$. Angiographic data obtained from cardiac catheterization laboratory records were randomly analyzed by at least two experienced clinicians who were blind to the study protocol. The left ventricular ejection fraction (LVEF) calculated by the modified Simpson method was rescanned using the hospital database. The diagnosis of acute myocardial infarction (AMI) including STEMI and NSTEMI was determined based on ESC criteria ${ }^{(6)}$. AMI was defined as elevated myocardial necrosis markers greater than the upper limit of the normal range, with either ischemic symptoms or changes in electrocardiographic or non-invasive stress images indicating ischemia. UA was defined as myocardial ischemia at rest or with minimal exertion, in the absence of myocardial necrosis. Hypertension was defined as a systolic blood pressure $\geq 140 \mathrm{mmHg}$ or a diastolic blood pressure $\geq 90 \mathrm{mmHg}$, or use of antihypertensive drugs ${ }^{(7)}$. Diabetes was defined as a fasting serum glucose level $\geq 126 \mathrm{mg} / \mathrm{dL}, \mathrm{HbA} 1 \mathrm{c} \geq 6.5 \%$ or being under treatment ${ }^{(8)}$.

\section{CAD Severity Scores}

Coronary lesion severity was determined using the SYNTAX I and Gensini scores. SYNTAX I score was 
calculated for stenosis diameter of $50 \%$ or greater in vessels of $1.5 \mathrm{~mm}$ or more in diameter and the final online updated version (2.11) was used (www.syntaxscore.com ${ }^{(9)}$. The Gensini score was calculated by assigning a severity score to each coronary lesion according to the degree of narrowing and geographical importance (stenosis of $25 \%, 50 \%, 75 \%, 90 \%$, and $99 \%$, and total occlusion were given as Gensini scores of 1, 2, 4, 8, 16, and 32 points, respectively; the importance of its localization, left anterior descending coronary artery (LAD) $\times 2.5$; the proximal segment of the circumflex artery $(\mathrm{LCX}) \times 2.5$; the mid-segment of the $\mathrm{LAD} \times 1.5$; the right coronary artery (RCA), the distal segment of the LAD, the posterolateral artery and the obtuse marginal artery $\times 1$; and others $\times 0.5$ ). The total score was equal to the sum of the stenosis score and location score ${ }^{(10)}$.

\section{Statistical Analysis}

All data were analyzed using SPSS v.17.0 for Windows (SPSS, Inc., Chicago, Ill., USA). Categorical variables were presented as frequencies and percentages, continuous variables were expressed as means \pm standard deviation. Normal distribution was tested using the KolmogorovSmirnov test. A Student's t-test or Mann-Whitney U test was performed to compare the continuous variables based on the normality distribution. Correlation analysis was performed with the Spearman's coefficient of correlation. Categorical values were compared by using $\chi^{2}$ test and $\mathrm{p}<0.05$ was considered statistically significant.

\section{Results}

The clinical data of the study subjects are summarized in Table 1. Age, LVEF, HbA1c and white blood cells (WBC) differed significantly between the groups. However, there was no significant difference in hypertension and diabetes incidence, smoking, fasting glucose, creatinine, hemogram, CRP and lipid parameters. Additionally, the TyG index and TG/HDL-C did not differ significantly between the groups (TyG index, $8.97 \pm 0.72$ vs $9.06 \pm 0.72$, $\mathrm{p}=0.533$; TG/HDL-C, $3.64 \pm 2.36$ vs $3.99 \pm 2.23$, $\mathrm{p}=0.395$ ).
In the comparison based on the median TyG index, the TyG index was calculated as $8.46 \pm 0.39$ and $9.56 \pm 0.51$ in those with low and high TyG index, respectively $(p<0.001)$. The subjects with high TyG index had an increased incidence of hypertension and diabetes, a high fasting glucose, HbA1c, total cholesterol, TG and TG/ HDL-C ratio. However, HDL-C levels were significantly lower in this group $(\mathrm{p}<0.05)$ (Table 1$)$.

In the ACS subgroup, there was no significant difference in TyG index and the ratio of patients with a high TyG index. Patients with UA had lower Gensini and SYNTAX I scores, and the patients with NSTEMI showed a higher SYNTAX I score. There was no significant difference in CAD severity scores in patients with and without STEMI (Table 2). In the correlation analysis, TyG index had a significant relationship with HbA1c, total cholesterol, HDL-C and TG/HDL-C, but there was no correlation between the TyG index and CAD severity scores (Table 3).

\section{Discussion}

In this study, we investigated the relationship between the TyG index and the presence and severity of CAD. We found that increased TyG index was more closely related to MetS components and that TyG index did not play an important role in $\mathrm{CAD}$ diagnosis and progression.

The researchers agree that IR contributes to plaque progression in atherogenesis by inducing apoptosis of macrophage, endolytic cells, and smooth muscle cells. However, the results of human studies on atherogenesis are inconsistent ${ }^{(11)}$. In a study evaluating IR with Homeostatic Model Assessment of Insulin Resistance (HOMA-IR), high IR was associated with increased CAC in Japanese men, but in another study, no association between HOMA-IR and CAC was observed in patients without clinical signs of $\mathrm{CAD}^{(12,13)}$. Recently, the role of the TyG index in CVD has been investigated. In one study, TyG index was an independent predictor of cardiovascular event risk in a healthy population ${ }^{(14)}$. In another study, Kim et al. ${ }^{(15)}$ showed a high TyG index in a healthy population 
Table 1. Baseline characteristics of subjects

\begin{tabular}{|c|c|c|c|}
\hline Based on ACS presence & $\begin{array}{l}\text { ACS group } \\
(n=83)\end{array}$ & $\begin{array}{l}\text { Control group } \\
(n=50)\end{array}$ & $\mathbf{p}$ \\
\hline Age (years) & $64.64 \pm 12.79$ & $58.50 \pm 12.28$ & 0.007 \\
\hline Males, n (\%) & $54(65)$ & $28(56)$ & 0.298 \\
\hline Hypertension, n (\%) & $42(51)$ & $26(52)$ & 0.876 \\
\hline Diabetes mellitus, n (\%) & $28(34)$ & $15(30)$ & 0.656 \\
\hline Current smoking, n (\%) & $28(34)$ & $14(28)$ & 0.475 \\
\hline LVEF (\%) & $47.81 \pm 9.87$ & $53.66 \pm 9.52$ & 0.001 \\
\hline Fasting glucose (mg/dL) & $147.13 \pm 71.18$ & $131.76 \pm 70.77$ & 0.229 \\
\hline $\mathrm{HbA1c}(\%)$ & $7.46 \pm 1.35$ & $9.71 \pm 2.30$ & 0.004 \\
\hline Creatinine (mg/dL) & $0.99 \pm 0.31$ & $1.00 \pm 0.162$ & 0.193 \\
\hline TChol (mg/dL) & $169.66 \pm 33.60$ & $181.74 \pm 40.58$ & 0.066 \\
\hline LDL-C (mg/dL) & $101.54 \pm 31.34$ & $105.04 \pm 34.07$ & 0.547 \\
\hline HDL-C (mg/dL) & $41.06 \pm 10.11$ & $43.76 \pm 11.34$ & 0.157 \\
\hline TG (mg/dL) & $160.08 \pm 72.47$ & $135.24 \pm 74.35$ & 0.062 \\
\hline Hemoglobin (g/dL) & $12.97 \pm 1.96$ & $15.21 \pm 11.14$ & 0.083 \\
\hline WBC (cells/ $\mu \mathrm{L})$ & $10.29 \pm 3.76$ & $9.02 \pm 0.91$ & 0.043 \\
\hline CRP (mg/dL) & $5.09 \pm 16.02$ & $3.25 \pm 5.41$ & 0.434 \\
\hline TyG index & $8.97 \pm 0.72$ & $9.06 \pm 0.72$ & 0.533 \\
\hline TG/HDL-C & $3.64 \pm 2.36$ & $3.99 \pm 2.23$ & 0.395 \\
\hline Based on median TyG index & Low, $(n=67)(<9.01)$ & High, $(n=66)(>9.01)$ & p-value \\
\hline Age (years) & $62.46 \pm 14.32$ & $62.20 \pm 11.40$ & 0.906 \\
\hline Males, n (\%) & $45(67)$ & $37(56)$ & 0.188 \\
\hline Hypertension, n (\%) & $27(40)$ & $41(62)$ & 0.012 \\
\hline Diabetes mellitus, n (\%) & $9(13)$ & $34(52)$ & $<0.001$ \\
\hline Current smoking, n (\%) & $26(39)$ & $16(24)$ & 0.071 \\
\hline LVEF (\%) & $51.25 \pm 9.85$ & $48.74 \pm 10.29$ & 0.153 \\
\hline Fasting glucose (mg/dL) & $109.42 \pm 22.76$ & $173.77 \pm 87.38$ & $<0.001$ \\
\hline $\mathrm{HbA} 1 \mathrm{c}(\%)$ & $6.24 \pm 0.74$ & $8.60 \pm 1.89$ & 0.012 \\
\hline Creatinine (mg/dL) & $1.12 \pm 1.04$ & $1.10 \pm 1.04$ & 0.406 \\
\hline TChol (mg/dL) & $167.70 \pm 35.66$ & $180.80 \pm 36.83$ & 0.039 \\
\hline LDL-C (mg/dL) & $103.49 \pm 31.40$ & $102.21 \pm 33.44$ & 0.820 \\
\hline HDL-C (mg/dL) & $44.58 \pm 11.16$ & $39.53 \pm 9.49$ & 0.006 \\
\hline TG (mg/dL) & $95.37 \pm 32.06$ & $194.53 \pm 71.81$ & $<0.001$ \\
\hline Hemoglobin (g/dL) & $14.17 \pm 10.11$ & $13.45 \pm 1.36$ & 0.568 \\
\hline 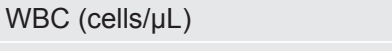 & $9.55 \pm 3.85$ & $10.08 \pm 3.13$ & 0.389 \\
\hline CRP (mg/dL) & $5.74 \pm 17.85$ & $3.03 \pm 4.49$ & 0.233 \\
\hline TyG index & $8.46 \pm 0.39$ & $9.56 \pm 0.51$ & $<0.001$ \\
\hline TG/HDL-C & $2.33 \pm 1.08$ & $5.23 \pm 2.32$ & $<0.001$ \\
\hline
\end{tabular}

LVEF: Left ventricular ejection fraction, TChol: Total cholesterol, LDL-C: Low-density lipoprotein cholesterol, HDL-C: High-density lipoprotein cholesterol, TG: Triglycerides, WBC: White blood cells, CRP: C-reactive protein, TyG index: Triglyceride glucose index, ACS: Acute coronary syndrome, n: Number 
Table 2. The clinic and angiographic data according to the presentation of acute coronary syndrome

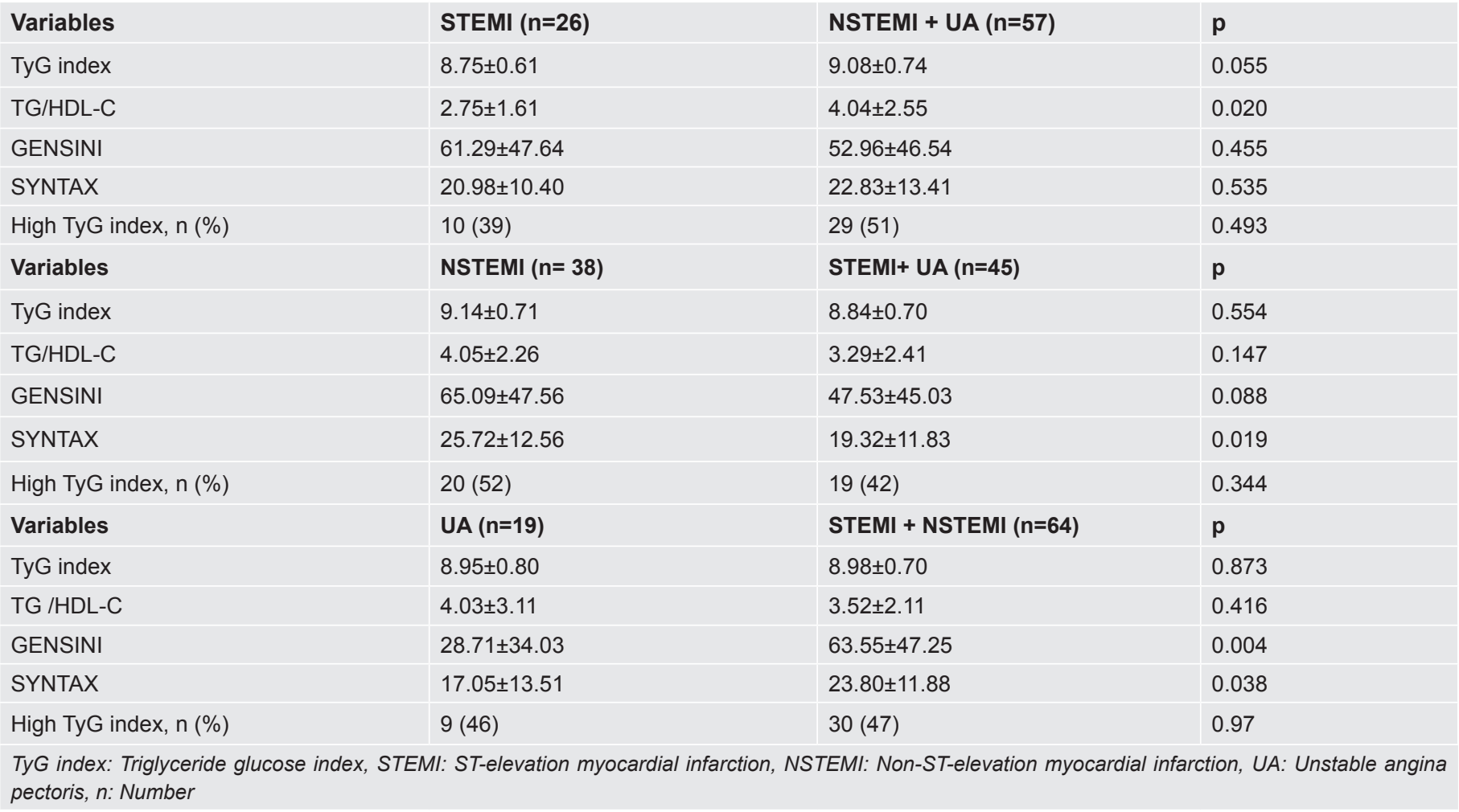

Table 3. Correlation analysis

\begin{tabular}{|l|l|l|}
\hline TyG index & $\mathbf{R}$ & $\mathbf{p}$-value \\
\hline Age & 0.044 & 0.616 \\
\hline LVEF & -0.060 & 0.490 \\
\hline HbA1C & 0.742 & $<0.001$ \\
\hline Creatinine & -0.015 & 0.865 \\
\hline TChol & 0.282 & 0.001 \\
\hline LDL-C & 0.034 & 0.627 \\
\hline HDL-C & -0.227 & 0.009 \\
\hline Hemoglobin & -0.016 & 0.856 \\
\hline WBC & 0.167 & 0,055 \\
\hline CRP & -0.041 & 0.636 \\
\hline TG/HDL-C & 0.706 & $<0.001$ \\
\hline Gensini & 0.902 & 0.406 \\
\hline SYNTAXI & 0.201 & 0.068 \\
\hline
\end{tabular}

TyG index: Triglyceride glucose index, LVEF: Left ventricular ejection fraction, TChol: Total cholesterol, LDL-C: Low-density lipoprotein cholesterol, HDL-C: High-density lipoprotein cholesterol, TG:

Triglycerides, WBC: White blood cells, CRP: C-reactive protein with increased CAC scores. Unlike these studies, there was no significant difference in TyG index between patients with and without ACS in our study. However, our study population consisted of patients with ACS and symptomatic subjects with at least one cardiometabolic risk factor and a high probability of CAD due to positive or suspected ischemia in non-invasive stress test. Also, in a study involving diabetic and non-diabetic patients, a high TyG index was associated with an increased risk of CAD only in healthy subjects, not in diabetic patients. Based on this study result, the importance of glucose control in diabetic patients was highlighted. It has also been claimed that atherogenic dyslipidemia in healthy subjects may affect subclinical atherosclerosis but chronic hyperglycemia exposure may be more effective in the development of atherosclerosis in diabetic patients ${ }^{(16)}$. In another study, Vega et al. ${ }^{(17)}$ also suggested that the TyG index may be a predictor of diabetes, which is a component of MetS rather than CVD. Also, the drug use may cause 
different study results. Da Silva et al. ${ }^{(18)}$ showed that the TyG index increased in symptomatic patients with at least one risk factor compared to CAD patients under treatment and claimed that this unexpected result may be due to uncontrollable cardiovascular risk factors in symptomatic patients. The higher HbAlc levels in control subjects due to inadequate therapy or lack of diabetes diagnosis in our study confirm the da Silva et al.'s ${ }^{(18)}$ study and this may eliminate the significant difference in the TyG index in our groups. In addition, visceral fat distribution, body mass index, gender, ethnicity, age and the prolonged exposure to cardiometabolic risk factors may alter the study results.

When we divided the subjects based on the median TyG index, we found that TyG index was significantly associated with hypertension, diabetes, total cholesterol, HbAlc and HDL-C predisposing to MetS. The TyG index was also correlated with TG/HDL-C, another parameter indicating IR. The correlation between the TyG index and MetS components was clearly demonstrated in a study similar to ours ${ }^{(18)}$.

Few studies have investigated the role of TyG index in determining the CAD severity. In a study, the TyG index increased in patients with a high SYNTAX I score on coronary angiography ${ }^{(19)}$. On the contrary, we could not find any relationship of TyG index with SYNTAX I and GENSINI scores. These different results of studies may be due to heterogeneity of age and gender distribution, the differences in clinical characteristics, study design, median TyG index, ethnic diversity and cardiovascular risk profiles of study populations. Also, dynamic changes in the TyG index or duration of exposure to dysmetabolic conditions may better determine the CAD severity than the presence of dysmetabolic conditions or a high TyG index. In addition, we could not find any difference in the TyG index of ACS subgroups. Similar to our study, a study did not show the relationship between the TyG index and SYNTAX I in ACS subgroups ${ }^{(20)}$. However, NSTEMI patients in our study had a higher SYNTAX I score than the other subgroups. The higher SYNTAX I score in NSTEMI patients may be explained by the advanced age of NSTEMI patients, the presence of more comorbidities and longer exposure to cardiometabolic disorders, as shown in previous studies ${ }^{(21)}$.

\section{Study Limitations}

Our study had some limitations. This study was retrospective and single-center study. The study results may not be generalized to whole population due to the relatively small sample size. Other limitations of the study were that TG and glucose levels were measured once, and body mass index or waist circumference and dietary habits were not recorded. In addition, the basal insulin levels were not measured olarak so the association between the TyG index and HOMA-IR was not evaluated. Finally, we could not ignore the effects of medication on study results.

\section{Conclusion}

TyG index may be more closely related to cardiometabolic disorders predisposing to CAD. Therefore, the TyG index should be used as a predictor in determining the cardiovascular risk profile rather than the diagnosis and progression of CAD. In addition, the TyG index may be used to determine appropriate therapy strategies in the control of cardiovascular risk factors. However, large multi-center studies are needed to evaluate the role of the TyG index in CAD.

\section{Ethics}

Ethics Committee Approval: This study was approved by Pamukkale University Faculty of Medicine Hospital Ethics Review Board in accordance with the Declaration of Helsinki (27.10.2020, Protocol No: 020/70506)

Informed Consent: The requirement for informed consent was waived due to the retrospective design of the study.

Peer-review: Internally and externally peer-reviewed.

\section{Authorship Contributions}

Surgical and Medical Practices: G.N., S.Ç.Ş., G.Ş., Concept: S.Ç.Ş., G.N., Design: S.Ç.Ş., Data Collection or Processing: G.N, G.Ş, S.Ç.Ş., M.Ş., Analysis or 
Interpretation: M.G.G., M.Ş., S.Ç.Ş, Literature Search: S.Ç.Ş., M.Ş., Writing: S.Ç.Ş.

Conflict of Interest: The authors declare that they have no conflict of interest.

Financial Disclosure: The authors declare no financial support by any grant or research sponsor and no competing financial interest.

\section{References}

1. Smith SC Jr, Jackson R, Pearson TA, et al. Principles for national and regional guidelines on cardiovascular disease prevention: a scientific statement from the World Heart and Stroke Forum. Circulation 2004;109:3112-21.

2. Lee EY, Yang HK, Lee J, et al. Triglyceride glucose index, a marker of insulin resistance, is associated with coronary artery stenosis in asymptomatic subjects with type 2 diabetes. Lipids Health Dis 2016;15:155.

3. Kim JH, Lee DY, Park SE, et al. Triglyceride glucose index predicts coronary artery calcification better than other indices of insulin resistance in Korean adults: the Kangbuk Samsung Health Study. Precision and Future Medicine 2017;1:43-51.

4. Wang L, Cong HL, Zhang JX, et al. Triglyceride-glucose index predicts adverse cardiovascular events in patients with diabetes and acute coronary syndrome. Cardiovasc Diabetol 2020;19:80.

5. Simental-Mendía LE, Rodríguez-Morán M, Guerrero-Romero F. The product of fasting glucose and triglycerides as surrogate for identifying insulin resistance in apparently healthy subjects. Metab Syndr Relat Disord 2008;6:299-304.

6. Roffi M, Patrono C, Collet JP, et al. 2015 ESC Guidelines for the management of acute coronary syndromes in patients presenting without persistent ST-segment elevation: Task Force for the Management of Acute Coronary Syndromes in Patients Presenting without Persistent ST-Segment Elevation of the European Society of Cardiology (ESC). Eur Heart J 2016;37:267-315

7. Chalmers J, MacMahon S, Mancia G, et al. 1999 World Health OrganizationInternational Society of Hypertension Guidelines for the Management of Hypertension. Guidelines sub-committee of the World Health Organization. Clinical and Experimental Hypertansion. 1999;21:1009-60.

8. American Diabetes Association. 2. Classification and Diagnosis of Diabetes: Standards of Medical Care in Diabetes-2019. Diabetes Care 2019;42(Suppl 1):13-28.
9. SYNTAX working group. SYNTAX score calculator. Available at http:// www.syntaxscore.com. Accessed May 20, 2012.

10. Gensini GG. A more meaningful scoring system for determining the severity of coronary heart disease. Am J Cardiol 1983;51:606.

11. Linton MF, Fazio S. Macrophages, inflammation, and atherosclerosis. Int J Obes Relat Metab Disord 2003;27:35-40.

12. Yamazoe M, Hisamatsu T, Miura K, et al. Relationship of Insulin Resistance to Prevalence and Progression of Coronary Artery Calcification Beyond Metabolic Syndrome Components: Shiga Epidemiological Study of Subclinical Atherosclerosis. Arterioscler Thromb Vasc Biol 2016;36:17038.

13. Lee KK, Fortmann SP, Fair JM, et al. Insulin resistance independently predicts the progression of coronary artery calcification. Am Heart J 2009;157:939-45.

14. Sánchez-IÍnigo L, Navarro-González D, Fernández-Montero A, PastranaDelgado J, Martínez JA. The TyG index may predict the development of cardiovascular events. Eur J Clin Invest 2016;46:189-97.

15. Kim MK, Ahn CW, Kang S, Nam JS, Kim KR, Park JS. Relationship between the triglyceride glucose index and coronary artery calcification in Korean adults. Cardiovasc Diabetol 2017;16:108.

16. Cho YR, Ann SH, Won KB, et al. Association between insulin resistance, hyperglycemia, and coronary artery disease according to the presence of diabetes. Sci Rep 2019;9:6129.

17. Vega GL, Barlow CE, Grundy SM, Leonard D, DeFina LF. Triglycerideto-high-density-lipoprotein-cholesterol ratio is an index of heart disease mortality and of incidence of type 2 diabetes mellitus in men. J Investig Med 2014;62:345-9.

18. Da Silva A, Caldas APS, Hermsdorff HHM, et al. Triglyceride-glucose index is associated with symptomatic coronary artery disease in patients in secondary care. Cardiovasc Diabetol 2019;18:89.

19. Mao Q, Zhou D, Li Y, Wang Y, Xu SC, Zhao XH. The Triglyceride-Glucose Index Predicts Coronary Artery Disease Severity and Cardiovascular Outcomes in Patients with Non-ST-Segment Elevation Acute Coronary Syndrome. Dis Markers 2019;2019:6891537.

20. Sulistyono T, Nughara T, Wasyanto $T$. Glucose trigliserides index as a predictor of severity of coronary artery assesesed with syntax score I in aciuye coronary syndrome patients. Acta Cardiologia Indonesiana 2020;6:127-34.

21. Steg PG, Goldberg RJ, Gore JM, et al. Baseline characteristics, management practices, and in-hospital outcomes of patients hospitalized with acute coronary syndromes in the Global Registry of Acute Coronary Events (GRACE). Am J Cardiol 2002;90:358-63. 